\title{
Traduire
}

Une eutre perspective sur $r$ tatadciction

Revue française de la traduction

$244 \mid 2021$

Des jeux et des mots

\section{La saga du shah. La grande aventure des échecs}

Dominic Michelin

\section{(2) OpenEdition}

\section{Journals}

Édition électronique

URL : https://journals.openedition.org/traduire/2340

DOI : 10.4000/traduire.2340

ISSN : 2272-9992

Éditeur

Société française des traducteurs

Édition imprimée

Date de publication : 15 juin 2021

Pagination : 73-80

ISSN : 0395-773X

\section{Référence électronique}

Dominic Michelin, « La saga du shah. La grande aventure des échecs », Traduire [En ligne], 244 | 2021

mis en ligne le 15 juin 2021, consulté le 01 juillet 2021. URL : http://journals.openedition.org/traduire/ 2340 ; DOI : https://doi.org/10.4000/traduire.2340 


\section{La saga du shah}

La grande aventure des échecs

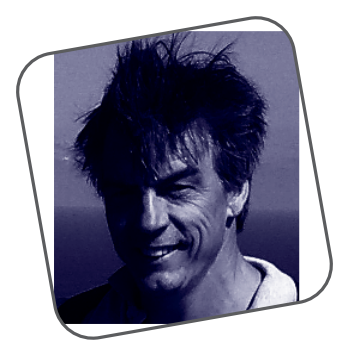

\section{Dominic Michelin}

\section{Origines, tribulations et consécration}

\section{"Once a pawn a time»}

Selon une très ancienne légende indienne, il était une fois un roi nommé Belkib qui s'ennuyait ferme dans l'exercice solitaire de son pouvoir absolu. Afin d'y remédier, il promit une récompense exceptionnelle à qui lui proposerait une distraction inédite pour le sortir de son enfermement et de la mélancolie que sa situation lui procurait. Se présenta alors un brahmane nommé Sissa qui lui dévoila un jeu destiné à démontrer d'une part la faiblesse et la vanité d'un monarque sans entourage et d'autre part la nécessité de demeurer humble. Ce jeu regroupait sur un plateau comportant soixante-quatre cases des figurines alignées représentant les quatre corps constituant jadis l'armée indienne traditionnelle: éléphants, cavaliers, chars et fantassins. Ainsi naquit le «chaturanga» ou «Jeu des quatre armes», impliquant stratégie et réflexion pour venir à bout de l'armée adverse. Le monarque fut séduit à un tel point par ce jeu qu'il souhaita remercier son inventeur en le laissant choisir lui-même sa récompense. Sissa réfléchit quelques secondes et demanda qu'on lui apportât une poignée de grains de blé. Il invita ensuite le souverain à placer un grain sur la première case du plateau, puis deux sur la deuxième case, quatre grains sur la troisième, huit sur la quatrième, et d'opérer ainsi jusqu'à 
la dernière case en doublant à chaque fois le nombre de grains pour obtenir le résultat final en volume de grains de blé qui lui serait versé. Cette demande sembla de prime abord bien modeste au souverain, si surpris et amusé par l'exercice qu'il accepta bien volontiers d'y répondre favorablement. Or le roi ne put jamais récompenser Sissa, car il lui aurait fallu offrir la bagatelle de 18446744073709551615 grains... soit l'intégralité de toutes les moissons de la Terre pendant environ 5000 ans! Et, en s'engageant à respecter sa promesse, il venait de condamner par la même occasion son royaume à la faillite. L'histoire ne dit pas ce qu'il advint réellement, mais le sage et sagace Sissa, en donnant cette leçon au roi, venait tout simplement de procéder à la genèse d'un jeu qui allait bientôt conquérir le monde et passer à la postérité.

$\mathrm{Ce}$ jeu de conception guerrière va rapidement évolver au cours de son périple planétaire sous l'influence des pays et cultures qui l'adoptent. Gagnant l'Occident via la Perse envahie par les Arabes qui l'y découvrent sous le nom de "chatrang» et en deviennent adeptes jusqu'à le faire remonter en Espagne via l'Afrique du Nord lors de leur conquête de la péninsule ibérique, il finit de coloniser le reste de l'Europe à la fin du $x^{e}$ siècle. Le jeu s'adapte en changeant de nom dans les différentes langues européennes qui reprennent phonétiquement le cri de victoire des armées perses: "Shah Mat» (littéralement, "le roi est mort»). II se structure peu à peu, adopte les cases de couleur alternée et se normalise avec l'invention de l'imprimerie qui contribue à sa diffusion. À la faveur de règles rigoureuses et d'une étonnante capacité d'adaptation culturelle et linguistique, notamment dans la représentation symbolique de ses pièces, il devient un jeu requérant à la fois rapidité d'exécution et qualité de réflexion.

À peine arrivé en Europe, ce nouveau jeu intrigue, et en moins d'un siècle, il envahit la société médiévale dans toutes ses couches de population. II subjugue l'aristocratie, qui transforme peu à peu ce divertissement d'origine guerrière en une distraction de cour qui reprend les valeurs de la société féodale et illustre les romans de chevalerie. Les pièces sont renommées, leur valeur reprécisée et leur pouvoir clairement spécifié. Une fois son cadre fixé et ses règles codifiées, l'échiquier se fait le symbole de la société nouvelle du Moyen Âge avec ses différentes catégories sociales. Les deux 
armées d'origine se transforment en véritables cours. Si le roi reste roi, le fantassin évolue en pion, le char devient tour, l'éléphant se fait fou, juge ou évêque selon les perceptions et les cultures, et le chevalier disparaît au profit de sa monture, tout en conservant son nom démocratisé en simple cavalier. Mais le changement essentiel survient avec l'arrivée ô combien emblématique de la reine comme unique élément féminin qui devient, excusez du peu, la pièce maîtresse du jeu, en remplacement du vizir ou conseiller originel.

De la tactique guerrière née sur le continent indien en proie aux conflits intérieurs à la compétition ludique de l'ère moderne, en passant par la distraction de cour de l'Occident médiéval ou encore le jeu de séduction induit par l'amour courtois, les échecs apparaissent comme le miroir du monde dans lequel ils passent, évolvent et s'installent, en en reflétant les valeurs sociales et culturelles, en en reprenant les concepts, principes, et par conséquent, les termes et expressions consacrées.

\section{Adaptation linguistique et évolution (multi)culturelle}

Au fil de son histoire depuis sa création, le jeu d'échecs a sans nul doute contribué à la communication interculturelle et donc multilingue, à transmettre et à adapter le vocabulaire inhérent au jeu et ainsi à favoriser les échanges entre peuples pour conquérir la planète entière et se faire langage universel.

Les échecs sont un langage international.

Emmanuel LASKER (1868-1941), $2^{\text {e }}$ champion du monde

L'intérêt du jeu d'échecs pour les linguistes et acteurs interculturels que nous sommes réside, entre autres, dans la traduction, l'évolution sémantique et l'adaptation du nom des pièces, dans sa terminologie spécifique et ses expressions idiomatiques qui ont fait leur petit bonhomme de chemin dans toutes les langues du monde. Pour illustrer ces propos, est compilé en annexe, à toutes fins utiles, un lexique trilingue français-anglais-espagnol des termes essentiels. Vous trouverez également nombre de clés terminologiques dans les sources citées. 
II y a plus d'aventure sur un échiquier

que sur toutes les mers du monde.

Pierre MAC ORLAN, in L'Ancre de miséricorde

Levons donc le rideau sur cet univers pour en découvrir la distribution et les fonctions de ses différents personnages, ainsi que ses rouages et arcanes grâce à un petit tour d'horizon historique et terminologique de ce théâtre si particulier qu'est l'échiquier.

Si, pour reprendre le contre-pied théâtral, le roi demeure dans toutes les langues indo-européennes avec son statut de monarque et ses racines latines, germaniques ou slaves, qu'il soit raja(h) sur son continent d'origine ou s(c)hah dans la sphère persane et turcophone, la reine est le plus souvent appelée dame, dénotant à la fois sa puissance et son indépendance affichées et souhaitées, et ce dès le Moyen Âge. En effet, sous l'influence des reines médiévales et de leur pouvoir souvent plus officieux qu'officiel, la pièce se féminise et acquiert sa primauté lors de sa première renaissance occidentale, même si - et c'est aussi intéressant à noter d'un point de vue culturel et linguistique - elle conserve par ailleurs sa dénomination et son statut de vizir ou de ministre en hindi, farsi, turc, arabe et même hongrois.

Quant à la tour, descendante du char ou chariot de guerre originel (roukh), l'arme légendaire des armées aryennes et pièce alors la plus puissante du jeu indien, elle s'est métamorphosée au cours de ses pérégrinations, mais conserve sa force symbolique, ainsi que ses origines indiennes et perses en anglais (rook) et en islandais (hrokur). Si elle ne s'appelle plus «roc» en français et a définitivement pris la dénomination de tour, voire de château dans presque toutes les autres langues, le terme originel a perduré dans certaines langues dont le français pour désigner le seul mouvement simultané autorisé impliquant deux pièces à la fois (en l'occurrence le roi et la tour), appelé "roque». D'ailleurs, noblesse oblige, ce mot désigne également en héraldique, le «meuble»représentant une tour.

La troisième pièce, le bien nommé fou - à égalité avec le cavalier par sa valeur -, constitue un autre bel exemple d'adaptation linguistique, voire de métamorphose culturelle. Éléphant au commencement, il s'est métamorphosé en évêque, juge ou bouffon (d'où "fou») à cause, dit-on, de 
la forme de ses grandes oreilles d'origine assimilée à une coiffe d'évêque ou de juge pour les uns, à un bonnet de bouffon pour les autres. Paradoxalement, l'allemand et le polonais en ont fait un coureur (Läufer, goniec) - de diagonale, à tout le moins -, à l'instar de la plupart des langues germaniques et scandinaves. L'anglais et l'islandais l'ont rapidement transformé en évêque, l'albanais et le bulgare en officier, la plupart des langues slaves mais aussi l'italien, l'espagnol, le catalan ont conservé, et pour ces dernières latinisé, ses racines éléphantines (alfil, alfiere). Le portugais s'est distingué de ses cousines latines en optant lui aussi pour le dignitaire ecclésiastique, tandis que d'autres langues comme le finnois, le croate et jusqu'à l'espéranto en ont fait un messager ou un coursier. C'est dire le chemin parcouru par ce pourfendeur de diagonales, pourtant peu destiné à cet exercice à l'origine, et dont la réputation n'est plus à faire, à en juger par l'expression consacrée.

Quant au chevalier d'origine, il a laissé place textuelle à sa seule monture comme référence quasi universelle, sauf en anglais, en finnois, en estonien et en slovène. Le français, à l'instar du tchèque, a aussi adopté le simple cavalier. L'allemand, les langues scandinaves, le serbe et le polonais en font, pour leur part, un «sauteur» (Springer, Skoczek, скакаy) - d'obstacles, s'agissant de la seule pièce du jeu à pouvoir franchir les autres. Et si j'osais dire ici que le naturel revient au galop, la palme adaptative et étymologique revient au hongrois qui l'appelle en toute simplicité huszár, évoquant le célèbre régiment de cavalerie qui a marqué l'histoire...

Terminons cette saga terminologico-ludique par ni le moindre ni le moins important en nombre: le pion. L'allemand et les langues scandinaves, dans une logique de continuité sociale, en ont fait un paysan ou fermier (Baver, bonde), le finnois un simple militaire, le hongrois un paysan ou un piéton. Partout ailleurs, il est resté fantassin ou simple soldat... Si l'on résume, les langues slaves ont conservé le combattant de première ligne, les langues germaniques et gaéliques l'ont reconverti en chantre de la ruralité, et les langues latines l'ont diversement assimilé comme humble voyageur ou travailleur pédestre. Or le pion, sous ses apparences modestes et vulnérables, est loin d'avoir un destin funeste tout tracé car, dès lors qu'il parvient à l'ultime case où il peut procéder à sa 
promotion, il se transforme en la pièce (roi excepté) que souhaite son détenteur, et peut donc prétendre à une destinée des plus grandioses sinon royale. Bel exemple d'évolution sociale, non?

Quant au jeu d'échecs en soi, sans surprise, il a conservé peu ou prou le nom originel selon la perception de shah et s'y décline allègrement en fonction des prononciations, pouvant de ce fait se comprendre dans quasiment toutes les langues. Le français a fini par établir un terme aujourd'hui si francisé qu'il en paraît indigène et, au passage, traduit bien l'idée principale, à savoir mettre le roi en échec, donc le condamner, l'empêcher de régner, sans forcément le tuer ouvertement. C'est dire l'ironie de l'histoire... De la même façon, l'anglais a aussi plutôt bien assimilé et apprivoisé le principe général avec ces jolies tournures que sont check et checkmate, aux nombreuses acceptions. Et que dire de l'espagnol qui est allé chercher et officialiser ajedrez et jaque, a priori respectivement issus de chatrang et shah, même si le lien scriptural n'est pas directement évident? On y perçoit ici les influences constitutives et assimilatrices du castillan. Un autre terme dans cette même langue, pourtant étymologiquement plus proche, à savoir escaques, n'a finalement pas retenu la plus grande adhésion et, bien que très peu usité, désigne aujourd'hui à la fois la case de l'échiquier et le jeu lui-même. Et quid de «damer le pion»? Autre jolie expression échiquéenne passée dans le langage courant pour signifier une victoire plutôt inattendue et imparable, synonyme de «coiffer au poteau». À noter que l'équivalent anglais peut être to checkmate someone, de même que l'espagnol poner a alguien en jaque mate, formulations qui reprennent l'une comme l'autre le contexte échiquéen.

Bref, de beaux exemples de terminologie adaptative et de transculturalité réussie qui constituent l'essence même de ce jeu et illustrent le cosmopolitisme de ce phénomène ludique autant que social qui voit principalement les termes de langues européennes s'imposer et se retrouver dans sa nomenclature officielle. 


\section{Conclusion}

Les échecs sont utiles à l'exercice de la faculté de penser et à celle de l'imagination.

LEIBNIZ

À y regarder - et écouter - de plus près, les échecs s'articulent véritablement autour d'un langage universel où nombre de termes sont similaires ou du moins très proches et intelligibles, a fortiori en situation de contexte ludique ou compétitif, ou la compréhension et la communication sont facilitées. Le jeu peut constituer en ce sens un excellent cadre d'apprentissage et d'application d'une langue étrangère, sans pour autant en rester là. En effet, la réflexion et la stratégie exigées par ce jeu recouvrent des propriétés étrangement similaires à la perception d'une langue/culture et induisent une application et une utilisation des connaissances qui ne sont pas sans rappeler une autre activité. Le fait de choisir et positionner un adjectif, un adverbe, un nom ou une expression idiomatique influe sur le style, la fluidité et la lisibilité d'un texte. À l'instar du judicieux et significatif assemblage des mots qui contribue à la forme et au fond d'un texte, la combinaison d'une suite de coups structure la stratégie et donne à une partie d'échecs originalité, beauté et intensité, ce qu'un juste agencement des mots apporte aussi en dynamisme, en élégance et en naturel à une traduction aboutie. Il est d'ailleurs surprenant de voir que le parallèle s'opère aussi avec l'évolution de l'intelligence artificielle dans un cas comme dans l'autre, où mémoires, ordinateurs et autres logiciels adaptés ne cessent d'être élaborés et développés.

Si l'origine exacte des échecs reste méconnue ou nébuleuse, aucun autre jeu n'a autant suscité d'écrits: toute une littérature technique, allégorique et symbolique y est consacrée et ne cesse d'être enrichie et développée. Théâtre sociologique illustrant les échanges entre différentes civilisations et la mutation des relations sociales, les échecs incarnent l'histoire, le voyage, l'évolution, les interactions et la communication intrinsèques à l'espèce humaine. Le cheminement culturel du jeu, de ses pièces et de ses règles atteste de sa capacité à adopter un vocabulaire propre à chaque langue tout en étant international, et à devenir un moyen de 
communication et de rapprochement par-delà les frontières linguistiques et culturelles.

À l'instar du processus de traduction, depuis l'appropriation du texte source jusqu'au rendu final du texte cible, en passant par les phases d'adaptation et de relecture, le jeu d'échecs, par ses innombrables combinaisons imaginatives, est une $œ u v r e$ de construction, de stratégie et de plaisir intellectuel faisant appel à la sagacité, à la créativité, au talent, et parfois au génie. Que ce soient la stratégie à élaborer et à suivre, le recul et la lucidité nécessaires à sa mise en œuvre, ce jeu souvent qualifié de sport ou de science, voire d'art, implique des propriétés cognitives qui ne sont pas sans rappeler celles spécifiques à l'exercice de traduction, voire d'interprétation, selon la vitesse d'exécution exigée. Enfin, comme d'autres activités, la traduction et les échecs sont fortement confrontés depuis longtemps déjà à l'intelligence artificielle. Or celleci remplacera-t-elle véritablement un jour l'intuition et la subtilité que peut apporter l'esprit humain, sa sensibilité et ses nuances?

\section{dommichelin@gmail.com}

Dominic Michelin est traducteur-interprète indépendant (anglais-espagnol $>$ français) depuis 2011. De formation initiale littéraire et linguistique, il a enseigné le français langue étrangère au Canada et en Australie avant de se spécialiser en commerce international, puis en communication internationale trilingue et en traduction. II a notamment été responsable communicationmarketing pour une grande entreprise aéronautique. Dominic est diplômé de l'université de Dijon-Bourgogne (master en stratégies de communication internationale, 2001), de l'université de Paris et de I'ISIT (master en industrie des langues et traduction spécialisée, 2010). 


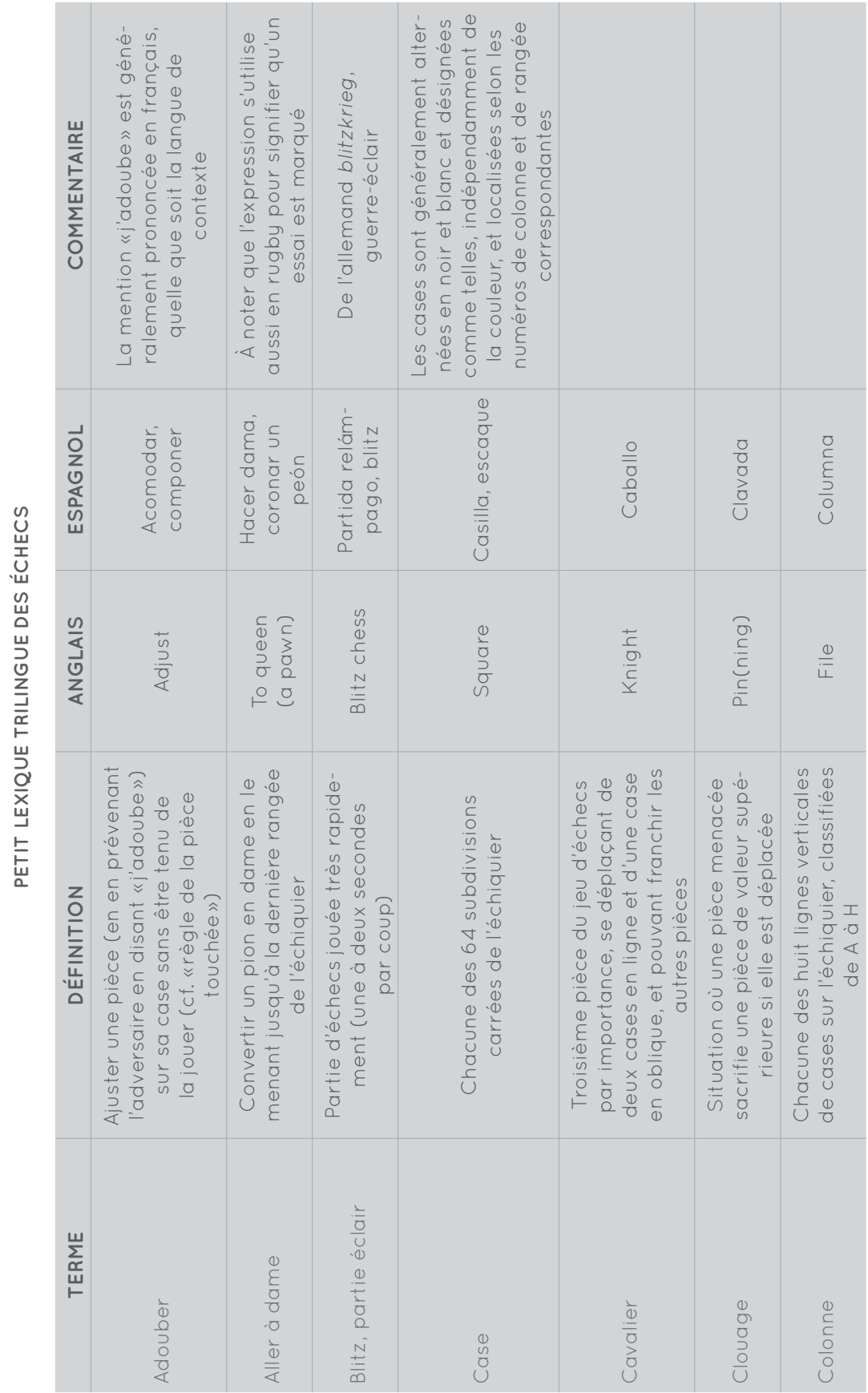




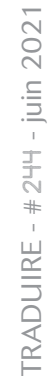

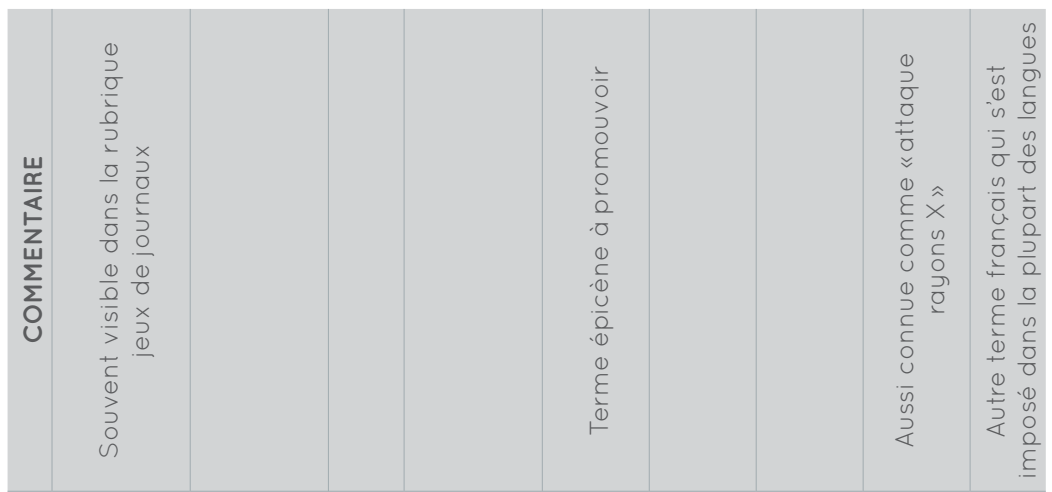

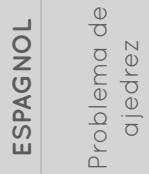

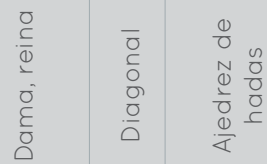

वे 0

8.

$\begin{array}{lll}\frac{0}{0} & \frac{0}{0} & \frac{0}{0} \\ \frac{0}{0} & \frac{1}{0} & \frac{0}{0} \\ 0 & \frac{1}{0}\end{array}$

$\frac{0}{2}$
$\frac{0}{0}$
$\frac{1}{5}$

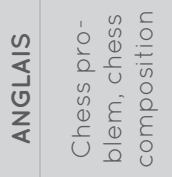

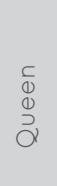

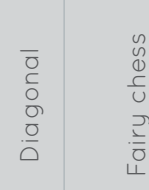

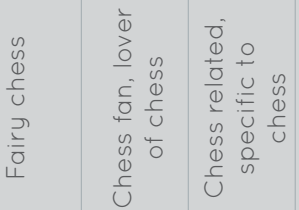

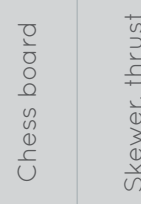

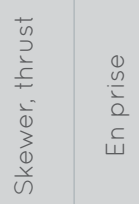

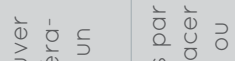

ว. ए)

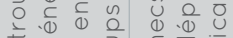

.

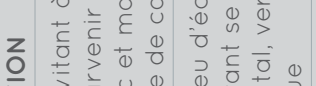

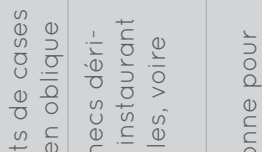

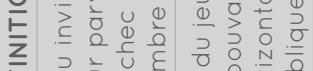

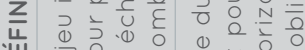

㟒

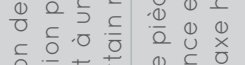

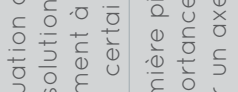

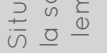

है है

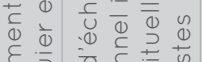

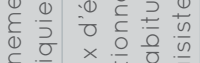

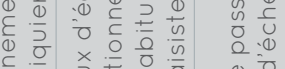

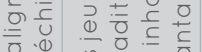

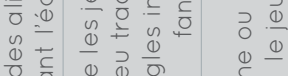

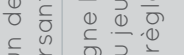

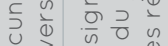

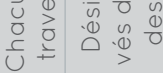

:.1)

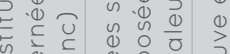

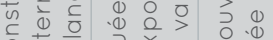

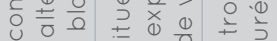

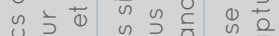

u)

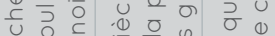

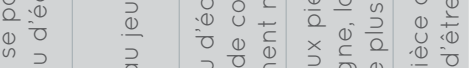

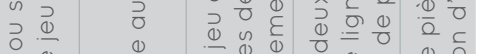

(1)

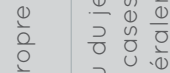

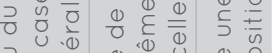

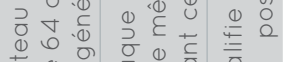

$\frac{\pi}{\square} \frac{0}{0}$

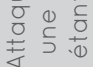
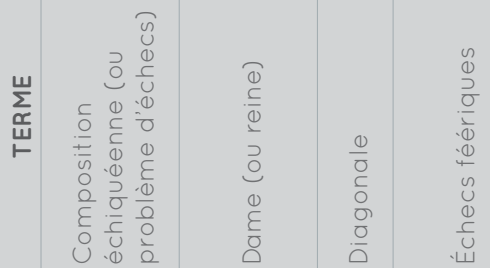

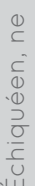

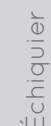

$\frac{1}{0}$
$\frac{1}{1}$
$\frac{2}{c}$
$\frac{1}{4}$ 


\begin{tabular}{|c|c|c|c|c|c|c|c|}
\hline 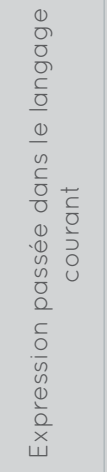 & & & & & 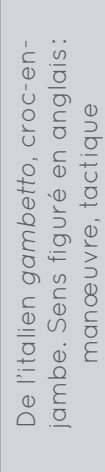 & & 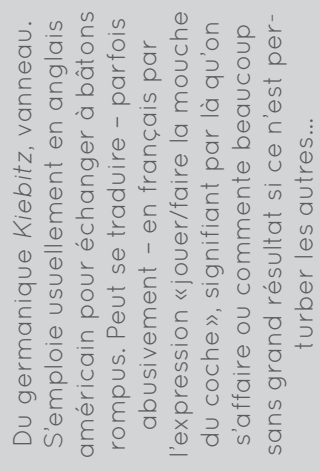 \\
\hline 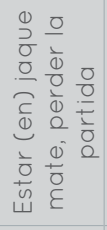 & 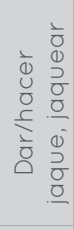 & 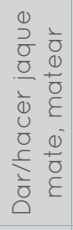 & $\overline{\bar{E}}$ & 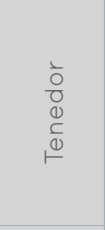 & 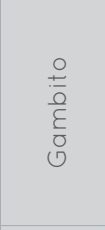 & $\begin{array}{l}0 \\
\frac{0}{0} \\
\frac{0}{0} \\
0 \\
\frac{1}{0} \\
0 \\
\frac{1}{4}\end{array}$ & 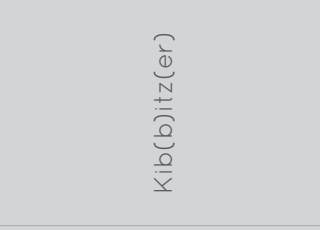 \\
\hline 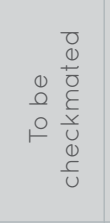 & 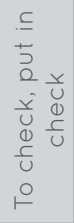 & $\begin{array}{l}0 \\
\frac{1}{0} \\
\xi \\
u \\
u \\
0 \\
\frac{1}{u} \\
\circ \\
\circ\end{array}$ & $\begin{array}{l}\frac{0}{0} \\
\frac{1}{n} \\
\frac{n}{0}\end{array}$ & $\begin{array}{l}\text { x } \\
\text { 후 }\end{array}$ & 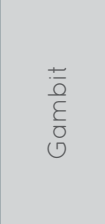 & 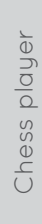 & 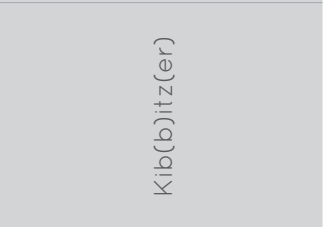 \\
\hline 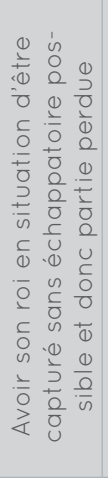 & $\begin{array}{l}0 \\
0 \\
\frac{1}{0} \\
0 \\
0 \\
0 \\
0 \\
0 \\
\frac{1}{0} \\
0 \\
0 \\
0 \\
0 \\
0 \\
0 \\
\overline{0} \\
0 \\
0 \\
\frac{1}{0} \\
0 \\
\Sigma\end{array}$ & 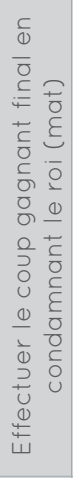 & 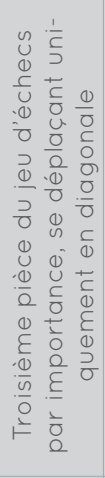 & 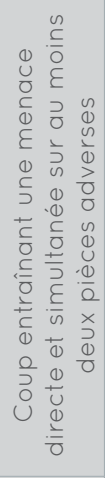 & 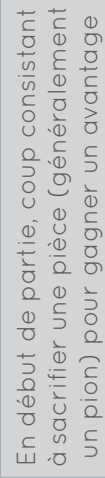 & & 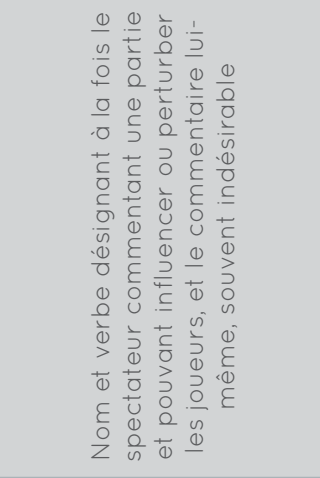 \\
\hline 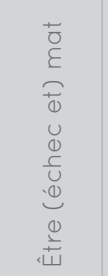 & 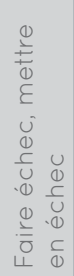 & 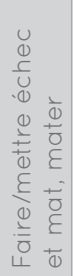 & $\begin{array}{l}\text { ?े } \\
\text { ४ }\end{array}$ & 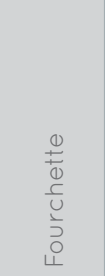 & $\begin{array}{l}\overline{0} \\
\bar{\varepsilon} \\
0 \\
0\end{array}$ & 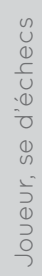 & 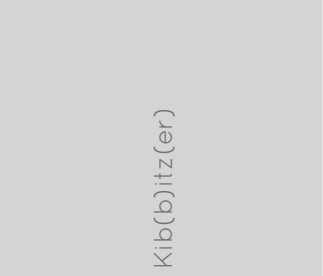 \\
\hline
\end{tabular}




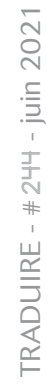

\begin{tabular}{|c|c|c|c|c|c|c|c|c|}
\hline 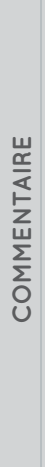 & & & 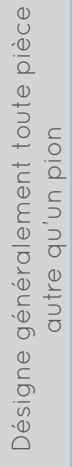 & 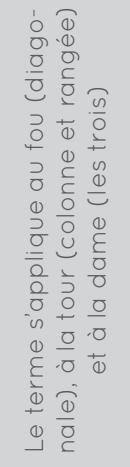 & & & 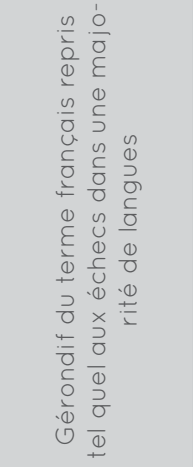 & \\
\hline 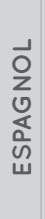 & $\begin{array}{l}\frac{1}{\partial} \\
\frac{0}{0} \\
\frac{0}{0} \\
\frac{1}{0} \\
\frac{1}{0} \\
\frac{1}{\Sigma}\end{array}$ & 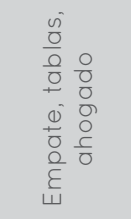 & $\begin{array}{l}\frac{0}{0} \\
0 \\
0 \\
\pm \\
\pm \\
0 \\
N \\
0 \\
0 \\
0\end{array}$ & 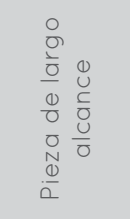 & 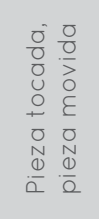 & $\begin{array}{l}c \\
0 \\
0 \\
0\end{array}$ & 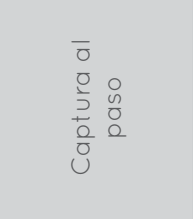 & $\begin{array}{l}\frac{1}{0} \\
\frac{1}{0} \\
\frac{N}{0} \\
\frac{0}{0} \\
0 \\
0 \\
\frac{0}{0} \\
\varepsilon \\
0 \\
0 \\
0\end{array}$ \\
\hline 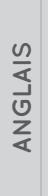 & 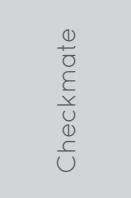 & $\begin{array}{l}\frac{0}{0} \\
\frac{\sigma}{0} \\
\frac{0}{0} \\
\dot{\sigma}\end{array}$ & 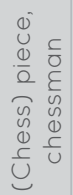 & 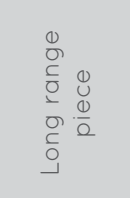 & 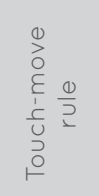 & $\begin{array}{l}5 \\
\vdots \\
0 \\
0\end{array}$ & $\begin{array}{l}\bar{c} \\
\overline{0} \\
0 \\
0 \\
0 \\
0 \\
0 \\
\text { c }\end{array}$ & $\begin{array}{ll} & \overline{0} \\
u & 0 \\
\omega & 0 \\
0 & 0 \\
\mathcal{L} & 0 \\
\cup & 0 \\
& 0\end{array}$ \\
\hline$\frac{z}{\underline{Z}}$ & 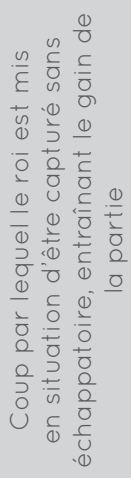 & 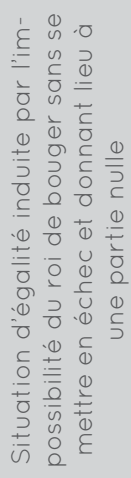 & 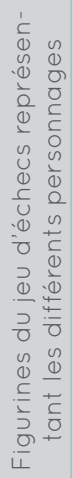 & 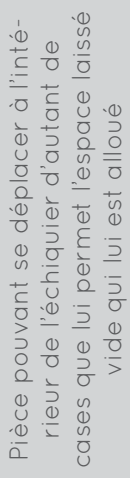 & 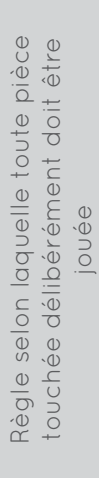 & $\begin{array}{l}0 \\
u \\
0 \\
\frac{1}{u} \\
0 \\
0 \\
0 \\
0 \\
\frac{0}{2} \\
0 \\
0 \\
0 \\
0 \\
0 \\
0 \\
0 \\
\frac{0}{0} \\
0 \\
0 \\
0 \\
\frac{0}{0}\end{array}$ & 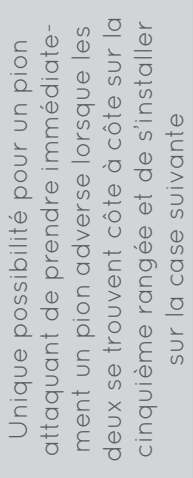 & 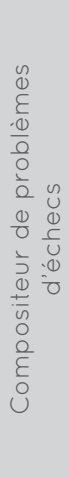 \\
\hline $\begin{array}{l}\underset{\sim}{w} \\
\underset{\leftarrow}{W}\end{array}$ & 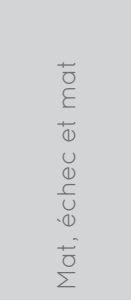 & 0 & $\begin{array}{l}0 \\
\stackrel{u}{0} \\
\stackrel{0}{\alpha}\end{array}$ & 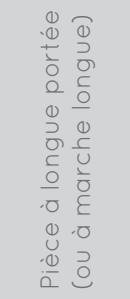 & $\begin{array}{l}0 \\
0 \\
0 \\
u \\
u \\
0 \\
0 \\
0 \\
0 \\
0 \\
0 \\
0\end{array}$ & $\frac{c}{0}$ & 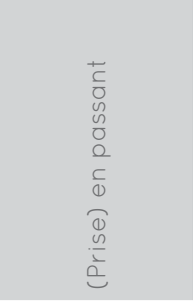 & $\begin{array}{l}\frac{0}{\frac{1}{0}} \\
\frac{\varepsilon}{0} \\
\frac{0}{0} \\
\frac{0}{0} \\
0\end{array}$ \\
\hline
\end{tabular}




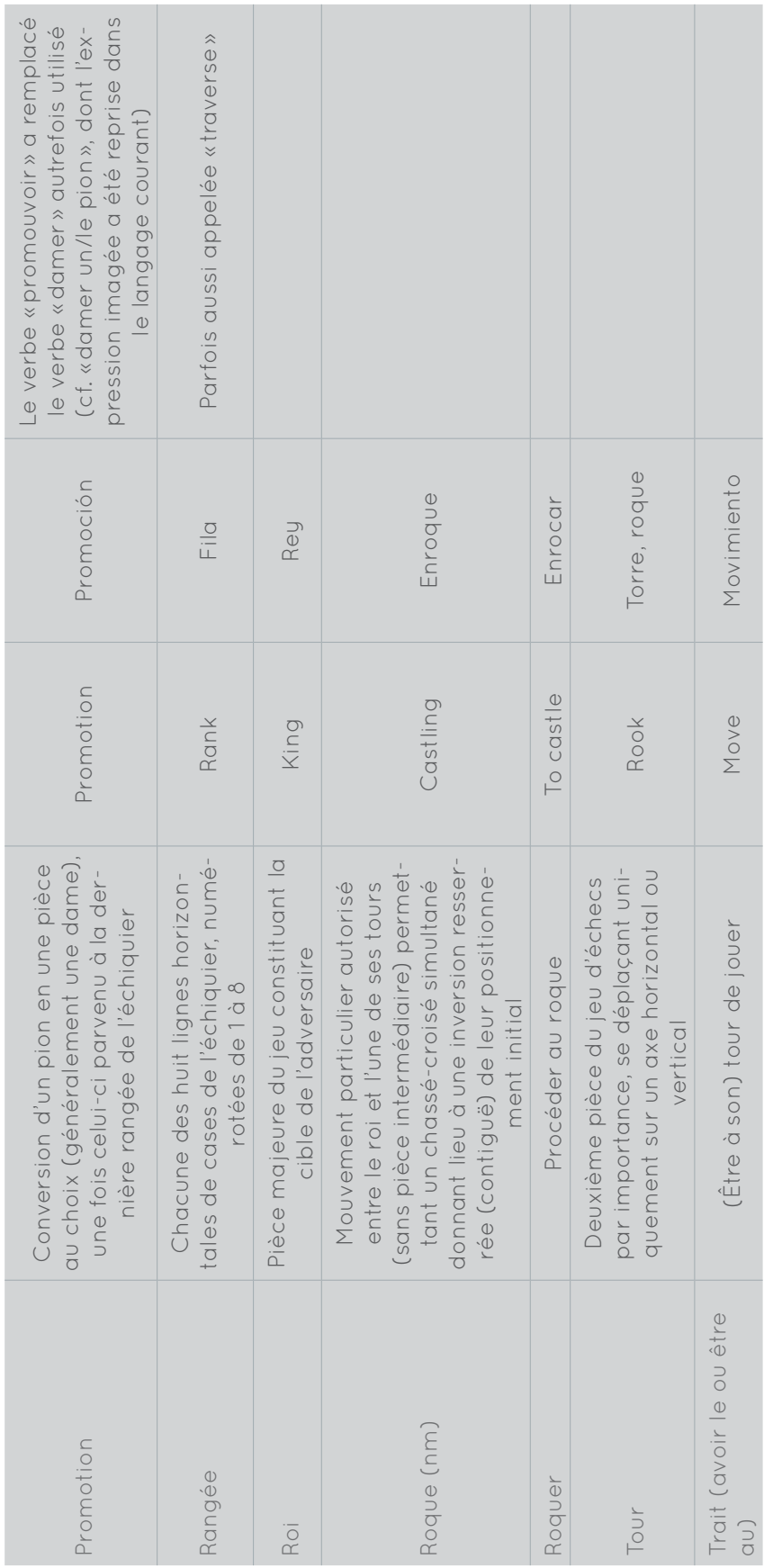

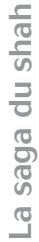


Ben Franklin, Blog, Chess.com, https://www.chess.com/blog/batgirl/ben-franklin, consulté le 28/04/2021.

École Apprendre-les-échecs, Le vocabulaire des échecs en anglais, https://ecole. apprendre-les-echecs.com/vocabulaire-echecs-anglais/, consulté le 28/04/2021.

Traduction de pièces d'échecs en langues internationales, https://www.schach-chess. com/jeu-echecs-pieces.htm, consulté le 28/04/2021.

Bnf, Le jeu des rois, http://classes.bnf.fr/echecs/acc/index.htm, consulté le 28/04/2021.

Pousseur de Bois, Le nom des pièces du jeu d'échecs, https://www.pousseurdebois.fr/ nom-des-pieces-aux-echecs/, consulté le 28/04/2021.

Alexa, Les origines du jeu d'échecs, source et histoire, Autour du jeu, 7 septembre 2017, http://autourdujeu.com/2017/09/07/les-origines-du-jeu-dechecs-source-et-histoire/, consulté le 28/04/2021.

Échecs, Le monde des échecs et de la culture, https://uia-echecs.blogspot.com/2014/10/ echecs-art-sciences-sport-jeu.html, consulté le 28/04/2021.

Chess24, Glosario, https://chess24.com/es/informate/glosario, consulté le 28/04/2021.

Wikipédia, Lexique du jeu d'échecs, https://fr.wikipedia.org/wiki/Lexique_du_ jeu_d\%27\%C3\%A9checs, consulté le 28/04/2021.

CHAN Amanda L., "Apprendre une langue étrangère : 7 raisons de parler une autre langue (ou plus)», The Huffington Post, 21 juin 2016, https://www.huffingtonpost.fr/2014/06/21/apprendre-langue-etrangere-7-raisons_n_5502798.html, consulté le 28/04/2021. 